\title{
Correction to: Facial nerve monitoring during parotid gland surgery: a systematic review and meta-analysis
}

\author{
Carlos Miguel Chiesa-Estomba ${ }^{1,8}$ (1) Ekhiñe Larruscain-Sarasola ${ }^{1} \cdot$ Jérome Rene Lechien $^{2,8} \cdot$ Francois Mouawad $^{3}$. \\ Christian Calvo-Henriquez ${ }^{4,8} \cdot$ Evelyne Siga Diom ${ }^{5} \cdot$ Adonis Ramirez $^{6} \cdot$ Tareck Ayad $^{7,8}$
}

Published online: 13 August 2020

๑) Springer-Verlag GmbH Germany, part of Springer Nature 2020

\section{Correction to: European Archives of Oto-Rhino-Laryngology \\ https://doi.org/10.1007/s00405-020-06188-0}

In the original publication of the article, the order of Figs. 2, 3 and 4 was published incorrectly.

The Fig. 2 was located as Fig. 4, Fig. 3 was located as Fig. 2 and Fig. 4 was located as Fig. 3.

The figures are placed in correct order in this correction.

The original article was updated.

The original article can be found online at https://doi.org/10.1007/ s00405-020-06188-0.

Carlos Miguel Chiesa-Estomba

chiesaestomba86@gmail.com

1 Otorhinolaryngology-Head and Neck Surgery Department,

Hospital Universitario Donostia, Calle Doctor

Begiristain, \#1. CP. 20014 San Sebastian-Donostia,

Guipuzkoa, Basque Country, Spain

2 Department of Human Anatomy and Experimental Oncology, University of Mons, Mons, Belgium

3 Department of Otorhinolaryngology-Head and Neck Surgery, CHRU de Lille, Lille, France

4 Department of Otolaryngology, Hospital Complex of Santiago de Compostela, Santiago de Compostela, Spain

5 ENT and Head and Neck Departement, CHU de La Paix, UFR Santé de l'Université Assane Seck, Ziguinchor, Sénégal

6 Head and Neck Surgery, Clinica Medilaser Neiva, Neiva, Colombia

7 Division of Otolaryngology-Head and Neck Surgery, Center Hospitalier de l'Université de Montréal, Montreal, Canada

8 Head and Neck Study Group of Young-Otolaryngologists of the International Federations of Oto-Rhino-Laryngological Societies (YO-IFOS), Paris, France 


\section{A Immediate}

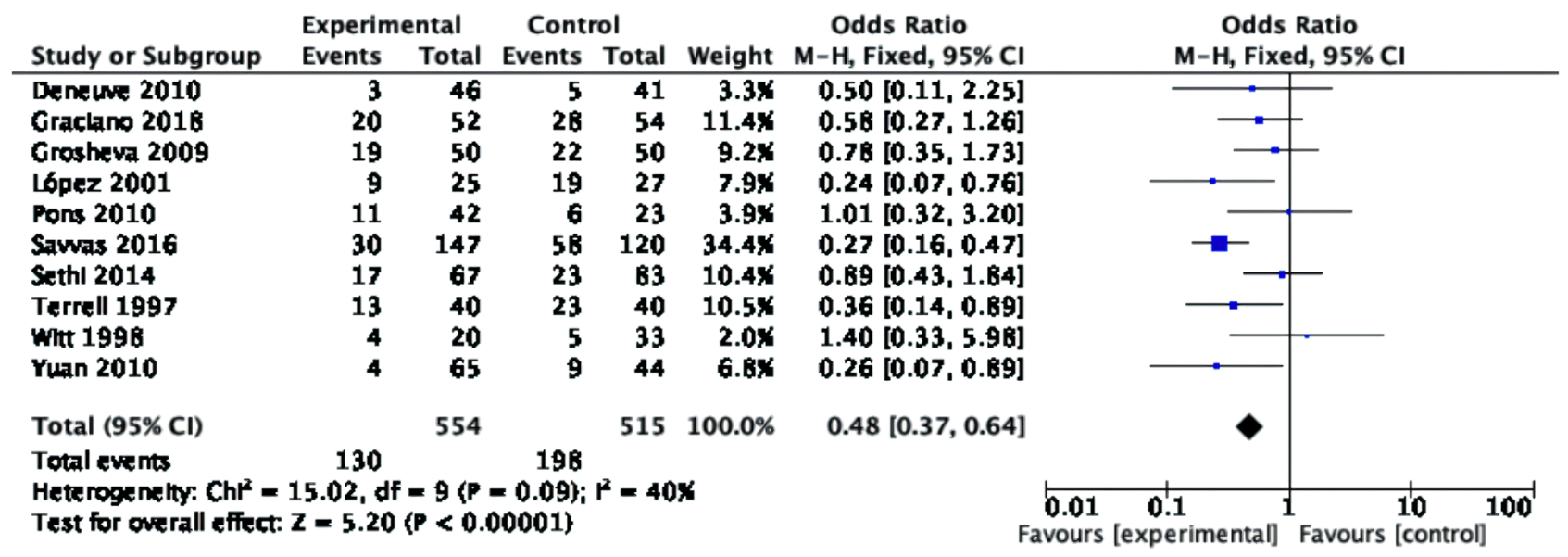

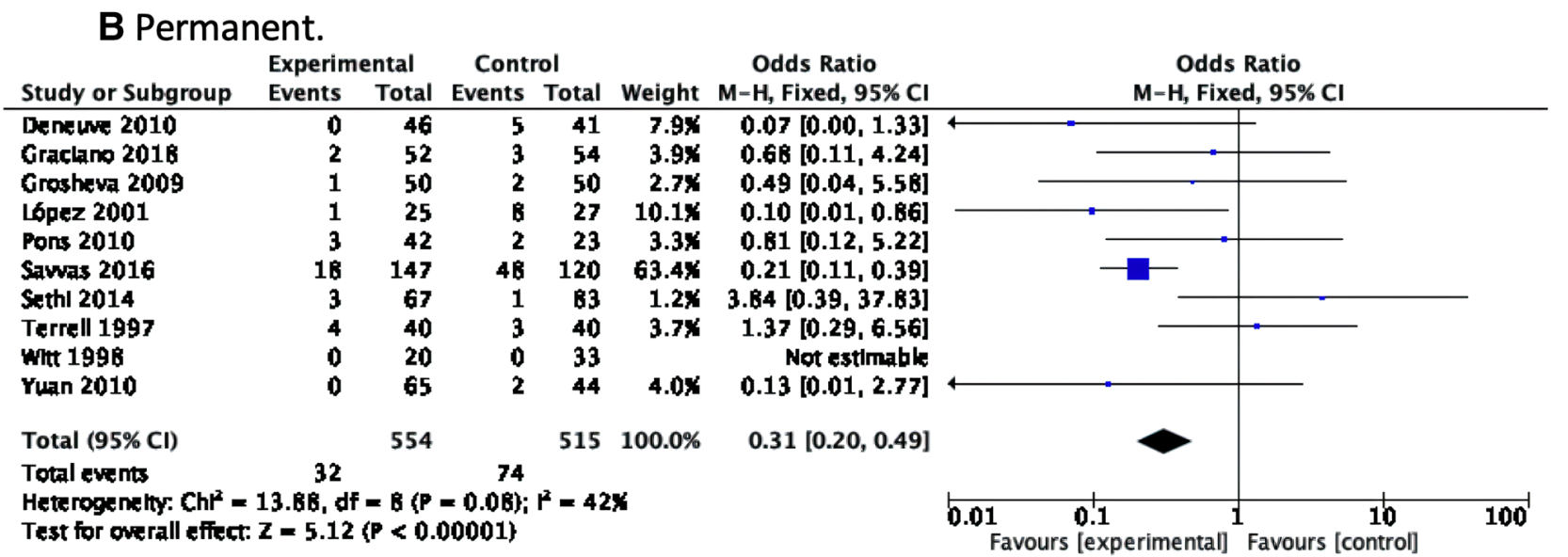

Fig. 2 Forest plot showing the rate of a immediate vs. b permanent facial nerve palsy. The experimental cohort (IFNM) vs. The control cohort (WIFNM) including all the studies 


\section{A Immediate:}

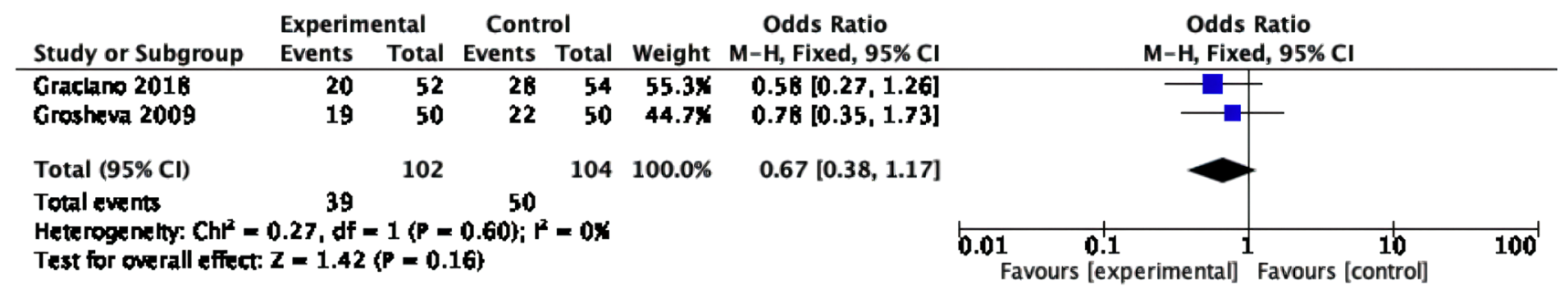

B Permanent:

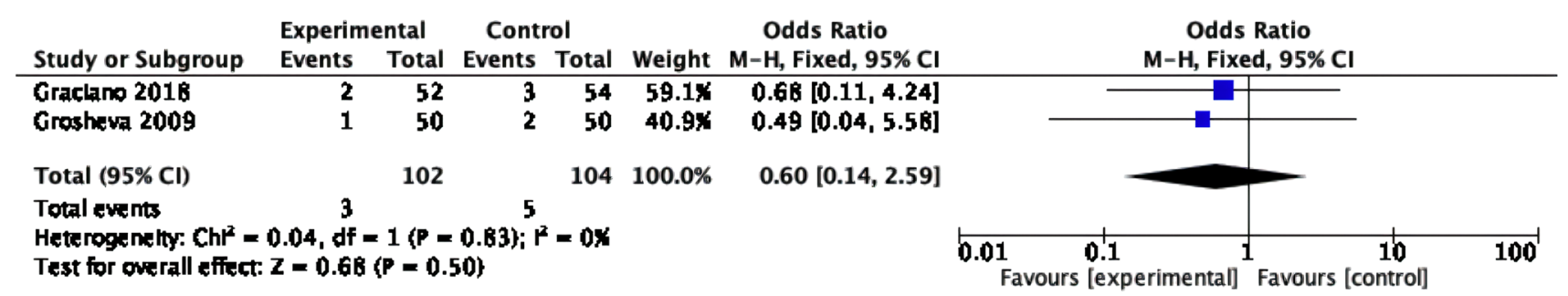

Fig. 3 Forest plot showing the rate of a immediate vs. b permanent facial nerve palsy. The experimental cohort (IFNM) vs. The control cohort (WIFNM) including just prospective data 


\section{Superficial parotidectomy}

A Immediate:

\begin{tabular}{|c|c|c|c|c|c|c|}
\hline Study or Subgroup & $\begin{array}{l}\text { Experim } \\
\text { Events }\end{array}$ & $\begin{array}{l}\text { ental } \\
\text { Total }\end{array}$ & $\begin{array}{r}\text { Cont } \\
\text { Events }\end{array}$ & $\begin{array}{l}\text { rol } \\
\text { Total }\end{array}$ & Weight & $\begin{array}{c}\text { Odds Ratio } \\
\text { M-H, Fixed, } 95 \% \mathrm{Cl}\end{array}$ \\
\hline Denelave 2010 & 2 & 20 & 2 & $1 \mathbf{B}$ & $2.2 \times$ & $0.89[0.11,7.06]$ \\
\hline Graclano 201B & 20 & 52 & $2 \mathbf{B}$ & 54 & $20.0 \%$ & $0.58[0.27,1.26]$ \\
\hline Grosheva 2009 & 12 & 41 & 16 & 38 & $13.9 \%$ & $0.57[0.22,1.44]$ \\
\hline 16pez 2001 & 5 & 17 & 9 & 16 & $7.7 \%$ & $0.32[0.08,1.36]$ \\
\hline Pons 2010 & 9 & 35 & 4 & 17 & $4.7 \times$ & $1.13[0.29,4.35]$ \\
\hline Sawas 2016 & 18 & 123 & 46 & 99 & $51.4 \%$ & $0.20[0.10,0.37]$ \\
\hline Total $(95 \% \mathrm{Cl})$ & & 288 & & 242 & $100.0 \%$ & $0.39[0.27,0.58]$ \\
\hline Total events & 66 & & 105 & & & \\
\hline
\end{tabular}

Heterogenelty: $\mathrm{ChP}^{2}=9.05, \mathrm{df}=5(\mathrm{P}=0.11) ; \mathrm{P}^{2}=45 \%$

Test for ove rall effect: $Z=4.79\langle P<0.00001\}$

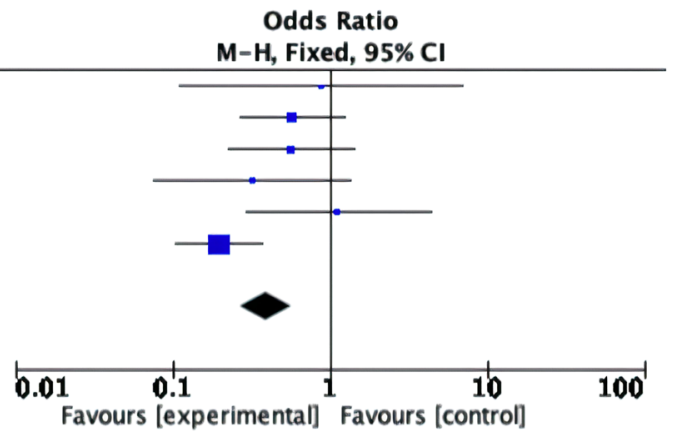

B Permanent:

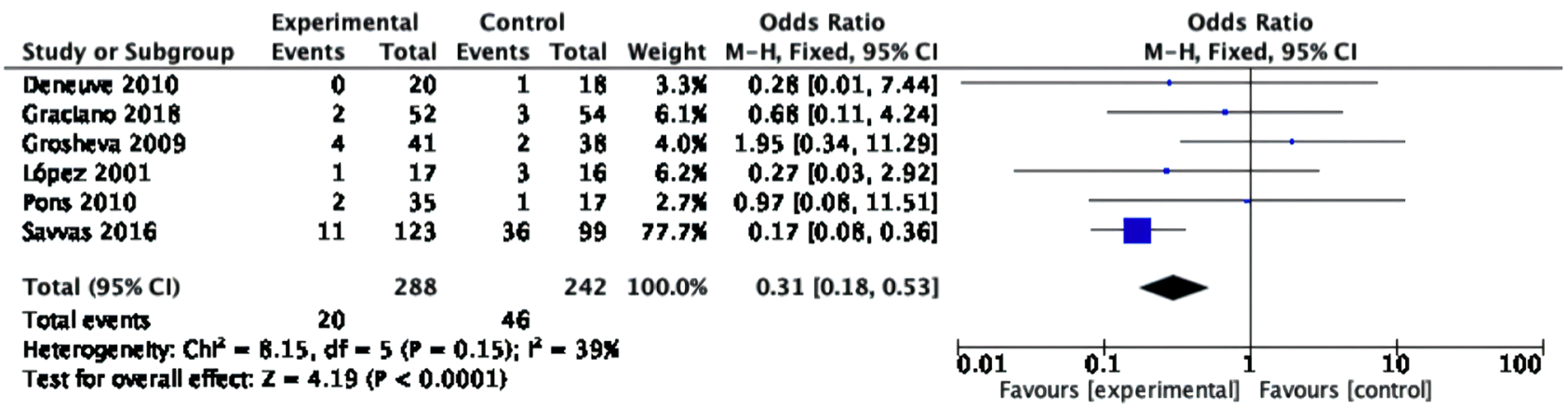

\section{Total parotidectomy}

A Immediate:

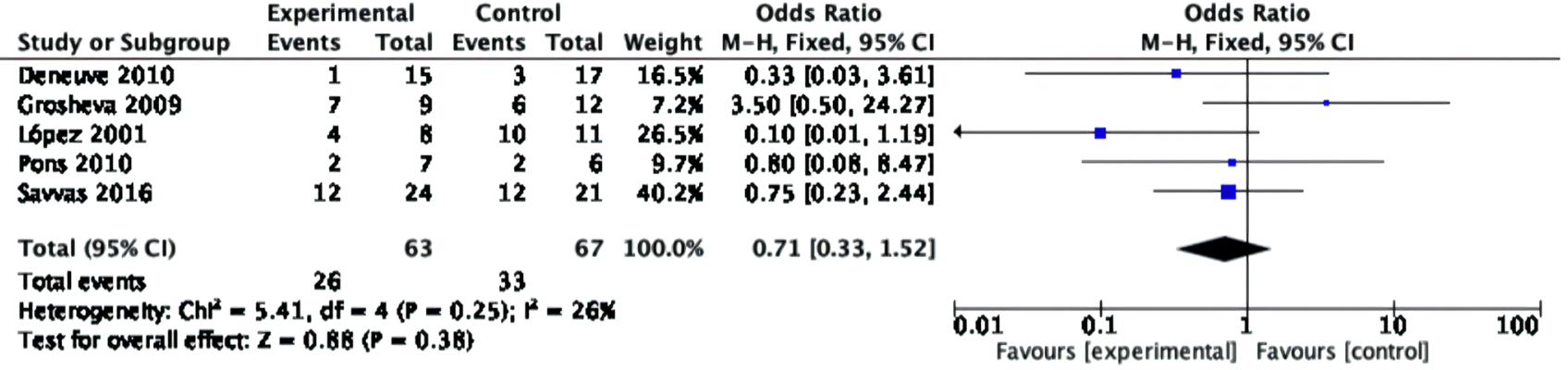

\section{B Permanent:}

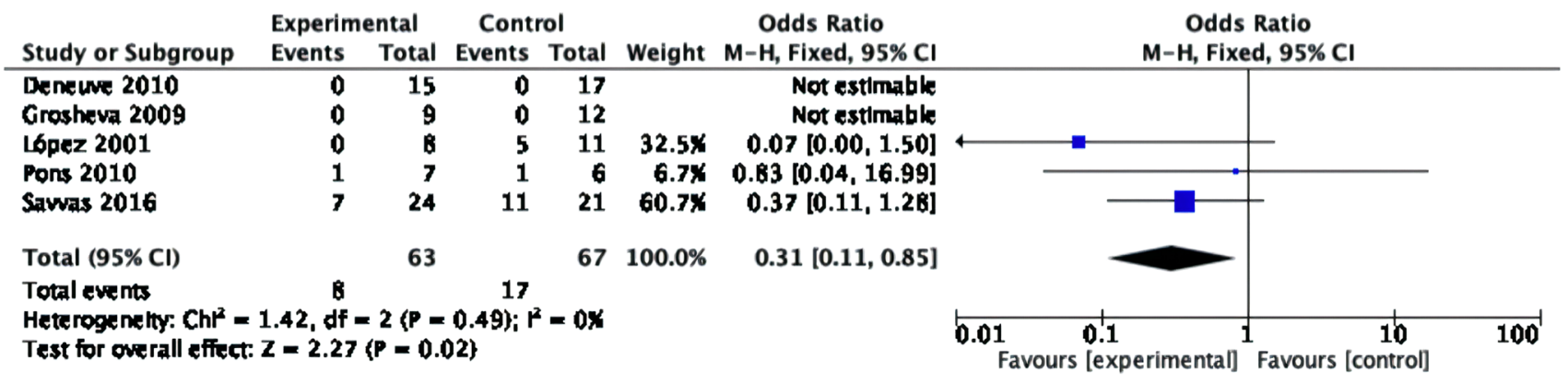

Fig. 4 Forest plot showing the rate of a immediate vs. b permanent Facial nerve palsy in patients underwent superficial and total parotidectomy. The experimental cohort (IFNM) Vs. The control cohort (WIFNM) 
Publisher's Note Springer Nature remains neutral with regard to jurisdictional claims in published maps and institutional affiliations. 